\title{
DI THƯỜNG VÀ BIẾN THIÊN THEO MÙA CỦA DÒNG ĐIỆN XÍCH ĐẠO
}

\author{
LÊ TRUOÒNG THANH ${ }^{1}$, LÊ HUY MINH ${ }^{1}$, HÀ DUYÊN CHÂU ${ }^{1}$, \\ DOUMOUYA VAFI ${ }^{2}$, YVES COHEN ${ }^{3}$ \\ E-mail: igpthanh@gmail.com \\ ${ }^{1}$ Viện Vật lý Địa cầu, 18 Hoàng Quốc Việt, Cầu Giáy, Hà Nội \\ ${ }^{2}$ Truòng Đại hoc tổng hợp Cocody, Coted'Ivoire \\ ${ }^{3}$ Viện Vật lý địa cầu Paris \\ Ngày nhận bài: 09-11-2010
}

\section{Mở đầu}

Các đặc trưng về cấu trúc, phân bố trong không gian và biến thiên theo thời gian của dòng điện xích đạo (Equatorial Electrojet - EEJ) phụ thuộc rất nhiều vào đặc trưng của trường địa từ vùng xích đạo, vào chu trình hoạt động Mặt trời. Để nghiên cứu những đặc tính đó có thể dựa vào các tài liệu quan trắc từ vệ tinh, tài liệu ghi biến thiên từ tại các đài trạm, tài liệu thăm dò điện ly thẳng đứng. Dựa vào số liệu trường từ ghi được tại các đài trạm nằm tại tâm của EEJ đã có nhiều công trình nghiên cứu về biến thiên theo thời gian của $\mathrm{EEJ}$ được công bố $[2,5,16]$ hoặc khi sử dụng số liệu thu được trên các vệ tinh và các số liệu khác $[1,3,11-14,17]$. Khi sử dụng số liệu trong khoảng thời gian ngắn thu được tại trạm Bạc Liêu của Việt Nam với các trạm nằm dọc theo xích đạo của Ân Độ, Châu phi...và số liệu trường từ thu được trên vệ tinh CHAMP, Doumouya V. [6] đã nhận thấy rằng cường độ $\mathrm{EEJ}$ tại kinh tuyến qua Việt Nam lớn hơn tại Ấn Độ và một số khu vực khác, nhưng do chuỗi số liệu còn ngắn nên chưa phản ánh được quy luật này có tồn tại và thay đổi theo mùa hay không. Trong [10] Le Mouël đã nghiên cứu biến thiên theo mùa của EEJ tính từ số liệu thu được trên vệ tinh CHAMP, nhưng mới chỉ sử dụng số liệu trong hai năm (2001-2002) và đây đúng vào năm mặt trời hoạt động mạnh. Trong [9] Kim H.R. cũng sử dụng số liệu của 57 tuyến số liệu thu được trên vệ tinh POGO để tìm ra những điểm dị thường EEJ trên toàn cầu, nhưng với chuỗi số liệu quá ít đã không phản ánh đúng vị trí các đỉnh này, và cũng không khẳng định được sự tồn tại của nó theo thời gian.

Trong bài báo trước [20] chúng tôi đã trình bày chi tiết về số liệu thu thập cũng như thuật toán để tách phần ảnh hưởng của $\mathrm{EEJ}$ từ số liệu trường từ thu được trên vệ tinh CHAMP và xây dựng mô hình EEJ trên toàn cầu dựa vào số liệu năm 2003, trong bài báo này, chúng tôi mở rộng chuỗi số liệu lên sáu năm liên tục (từ năm 2002-2007) đề nghiên cứu về các dị thường $E E J$ trên toàn cầu và biến thiên của nó theo thời gian và sau đó so sánh kết quả này với $\mathrm{EEJ}$ tính từ số liệu thu được tại các đài trạm mặt đất để bước đầu đưa ra những nhận xét về sự tồn tại các đỉnh dị thường và các đặc trưng biến thiên có chu kỳ của EEJ.

\section{Xử lý số liệu và phương pháp tách EEJ}

\subsection{Tính EEJ tù̀ số liệu vệ tinh CHAMP}

Trong nghiên cứu trước [20] chúng tôi đã giới thiệu về các thông số chính của vệ tinh CHAMP (quỹ đạo, độ cao,...) và số liệu thu được trên vệ tinh này, sau đó đã sử dụng số liệu thu được năm 2003 và áp dụng thuật toán để tách phần ảnh hưởng của $\mathrm{EEJ}$ từ số liệu trường từ thu được để nghiên cứu về EEJ và xây dựng mô hình $E E J$ trên toàn cầu. Để phục vụ cho nghiên cứu này, chúng tôi áp dụng thuật toán trên cho sáu năm số liệu liên tục thu được trên vệ tinh CHAMP. Hình $1 a$ trình bày kết quả ví dụ tính biên độ thành phần nằm ngang do hệ dòng EEJ gây ra cho số liệu thu được năm 2002 trên vệ tinh CHAMP khi sử dụng trường từ thành phần nằm ngang $(\mathrm{H})$ của 167 ngày trường 
từ yên tĩnh. Theo kết quả tính cả sáu năm số liệu, biên độ của EEJ thu được trên vệ tinh CHAMP biến đổi trong khoảng từ $20-60 n T$ và bề rộng ảnh hưởng của EEJ theo vỹ tuyến trong khoảng $\pm 8^{\circ}$.

\subsection{Tính EEJ tù̀ số liệu các trạm mặt đất}

Tương ứng với số liệu thu được trên vệ tinh CHAMP theo thời gian, chúng tôi lựa chọn số liệu thu được tại các trạm dưới mặt đất với những ngày trường yên tĩnh (chỉ số $a m<20$ ) với khoảng thời gian tương ứng. Để tách phần ảnh hưởng của $\mathrm{EEJ}$ từ số liệu trường từ thu được tại các trạm dựa vào giả thiết sau: dòng điện xích đạo như là một dải dòng hẹp có bề rộng khoảng $\pm 5^{\circ}$ chạy dọc theo xích đạo từ, trường từ của một trạm nằm cách xa tâm xích đạo từ khoảng $>10^{\circ}$ là coi như không bị ảnh hưởng của hệ dòng $\mathrm{EEJ}$ và trường từ thu được tại các trạm trên cùng một kinh tuyến, tại cùng thời gian do các hệ dòng khác có nguồn ở xa có thể coi như là hằng số. Như vậy, ta có thể sử dụng số liệu của một cặp trạm, bao gồm: một trạm nằm tại tâm của EEJ còn trạm kia có khoảng cách theo vỹ tuyến đủ xa để trường thu được không bị ảnh hưởng bởi hệ dòng EEJ và từng cặp trên trạm phải trên cùng một kinh tuyến, cùng có số liệu trong khoảng thời gian tính. Trong bài báo này, để so sánh EEJ thu được trên CHAMP và từ số liệu mặt đất chúng tôi sử dụng số liệu trên cùng một thời gian tại sáu trạm cho ba khu vực như trên hình $1 \mathrm{a}$, tên, ký hiệu và vị trí các trạm được trình bày trong bảng 1 (tọa độ địa từ tính theo niên đại 2005.0).

Với lựa chọn ba cặp trạm trên thì khoảng cách theo vỹ tuyến giữa FUQ-HUA là $17.97^{\circ}$, QSB-AAE là $24.85^{\circ}, \mathrm{PHU}-\mathrm{BCL}$ là $11.92^{\circ}$ và độ lệch giữa hai trạm trong từng cặp trạm theo kinh tuyến trong khoảng $3^{\circ}$.

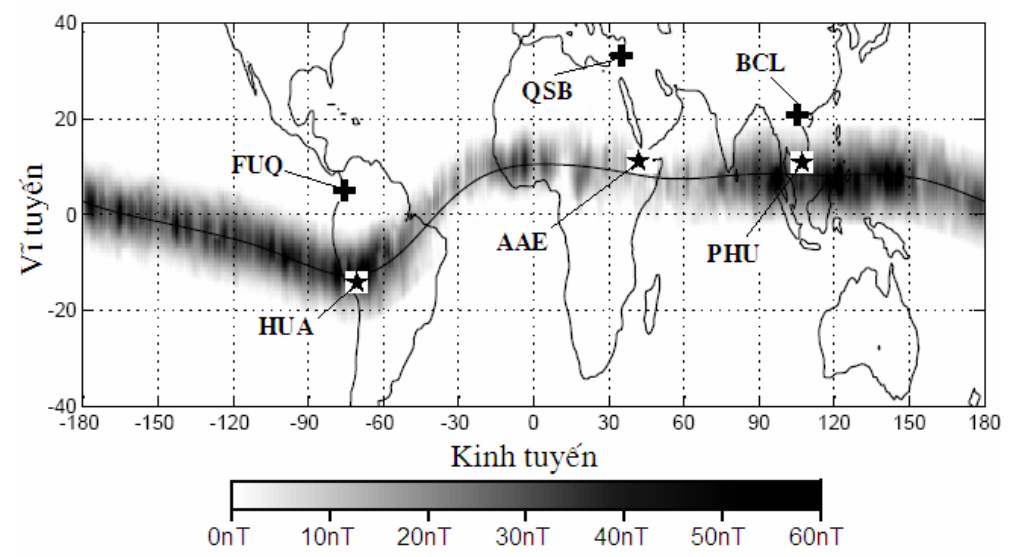

$\leftarrow$ Hình $1 a$. Giá trị trường từ thành phần nằm ngang do EEJ gây ra từ số liệu thu được trên vệ tinh CHAMP cho những ngày trường từ yên tĩnh năm 2002 và vị trí các đài trạm để nghiên cứu (hình ngôi sao là các trạm nằm gần tâm $\mathrm{EEJ}$, hình dấu cộng là các trạm nằm xa $\mathrm{EEJ}$ )

Bảng 1 . Vị trí và tên các đài trạm

\begin{tabular}{|c|c|c|c|c|}
\hline $\begin{array}{l}\text { Khu } \\
\text { vực }\end{array}$ & $\begin{array}{l}\text { Tên trạm và } \\
\text { ký hiệu }\end{array}$ & $\begin{array}{l}\text { Kinh độ } \\
\text { địa lý }\end{array}$ & $\begin{array}{l}\text { Vỹ độ } \\
\text { địa lý }\end{array}$ & $\begin{array}{l}\text { Vỹ độ } \\
\text { địa từ }\end{array}$ \\
\hline \multirow{2}{*}{$\begin{array}{l}\text { Khu } \\
\text { vực } \\
\text { châu } \\
\text { Á }\end{array}$} & $\begin{array}{l}\text { Bạc Liêu } \\
\text { (BCL) }\end{array}$ & $105.73^{\circ}$ & $9.28^{\circ}$ & $1.35^{\circ}$ \\
\hline & $\begin{array}{l}\text { Phú Thụy } \\
\text { (PHU) }\end{array}$ & $105.57^{\circ}$ & $21.2^{\circ}$ & $15.05^{\circ}$ \\
\hline \multirow{2}{*}{$\begin{array}{l}\text { Khu } \\
\text { vực } \\
\text { châu } \\
\text { Mỹ }\end{array}$} & $\begin{array}{l}\text { Huancayo } \\
\text { (HUA) }\end{array}$ & $284.67^{\circ}$ & $-12.5^{\circ}$ & $-1.61^{\circ}$ \\
\hline & $\begin{array}{l}\text { Fuquence } \\
\text { (FUQ) }\end{array}$ & $286.27^{\circ}$ & $5.47^{\circ}$ & $15.92^{\circ}$ \\
\hline \multirow{2}{*}{$\begin{array}{l}\text { Khu } \\
\text { vực } \\
\text { châu } \\
\text { Phi }\end{array}$} & $\begin{array}{c}\text { Addis Ababa } \\
\text { (AAE) }\end{array}$ & $38.77^{\circ}$ & $9.02^{\circ}$ & $5.27^{\circ}$ \\
\hline & $\begin{array}{c}\text { Qsaybeh } \\
\text { (QSB) }\end{array}$ & $35.64^{\circ}$ & $33.87^{\circ}$ & $30.23^{\circ}$ \\
\hline
\end{tabular}

Trong quá trình tính toán, chúng tôi sử dụng giá trị trung bình giờ của thành phần trường nằm ngang $\Delta \mathrm{H}$ của từng trạm toàn bộ số liệu sau khi đã loại bỏ giá trị trung bình giữa đêm. Khi ta coi trường do hệ dòng $\mathrm{Sq}$ gây ra tại từng cặp trạm trên là bằng nhau thì giá trị của thành phần nằm ngang $\mathrm{H}$ do EEJ gây ra tại ba trạm được tính như sau:

$$
\begin{aligned}
& \Delta \mathrm{H} \text { _EEJ_(hua) }=\Delta \mathrm{H} \text { (hua) }-\Delta \mathrm{H} \text { (fuq) } \\
& \Delta \mathrm{H} \text { _EEJ_(aae) }=\Delta \mathrm{H}(\text { aae })-\Delta \mathrm{H} \text { (qsb) } \\
& \Delta \mathrm{H} \text { _EEJ_(bcl) }=\Delta \mathrm{H}(\mathrm{bcl})-\Delta \mathrm{H}(\mathrm{phu})
\end{aligned}
$$

Với: $\Delta \mathrm{H}$ (hua) là giá trị thành phần nằm ngang $(\Delta \mathrm{H})$ tại trạm Huancayo sau khi đã trừ đi mức giữa đêm và tương ứng cho các trạm khác.

$\Delta$ H_EEJ_(hua) là giá trị thành phần nằm ngang $(\Delta \mathrm{H})$ do EEJ gây ra thu được tại Huancayo và tương ứng cho các trạm khác. Tuy nhiên trong thực tế, trường từ do hệ dòng $\mathrm{Sq}$ gây ra không những phụ thuộc vào thời gian mà còn phụ thuộc vào vị 
trí địa lý của từng trạm. Do đó, trường $\mathrm{Sq}$ thu được tại từng trạm trong từng cặp trạm là khác nhau, để loại bỏ ảnh hưởng này chúng tôi đưa vào một giá trị hiệu chỉnh ( $\mathrm{Sq}$ hc) khi sử dụng mô hình $\mathrm{CM} 4$ do Sabaka [18] phát triển. Mô hình cho phép tính trường từ do hệ dòng $\mathrm{Sq}$ gây ra tại từng vị trí dựa vào tham số dòng hạt mặt trời $(\mathrm{F} 10.7 \mathrm{~cm})$ tại từng vị trí và từng thời điểm khác nhau. Khi đó giá trị hiệu chỉnh $\mathrm{Sq}$ hc cho từng vị trí trạm được tính như sau:

$$
\begin{aligned}
& \text { Sq_hc_(hua) }=\mathrm{Sq} \text { CM4 _hua - Sq_CM4_fuq } \\
& \text { Sq_hc_(aae) }=\text { Sq_CM4_aae -Sq_CM4_qsb } \\
& \text { Sq_hc_(bcl) }=\text { Sq_CM4_bcl - Sq_CM4_phu }
\end{aligned}
$$

Trong đó: $\mathrm{Sq} C \mathrm{CM} 4$ hua là giá trị trường $\mathrm{Sq}$ tính theo mô hình tại trạm Huancayo, và tương ứng cho các trạm khác.

Sq hc (hua) là giá trị để hiệu chỉnh sự chênh lệch do dòng Sq gây ra tại trạm Huancayo và tương tự cho các trạm khác. Trên hình $1 \mathrm{~b}$ biểu diễn hệ dòng $\mathrm{Sq}$ tính theo mô hình $\mathrm{CM} 4$ phụ thuộc vào vĩ độ tại kinh tuyến đi qua trạm Bạc Liêu tính cho số liệu thu được vào lúc giữa trưa địa phương ngày 3-1-2005 và giá trị hiệu chỉnh $\mathrm{Sq}$ hc_(bcl) do ảnh hưởng của vị trí giữa trạm Phú Thụy và Bạc Liêu là $19 \mathrm{nT}$, tương tự: giữa Huancayo và Fuquence là $21 \mathrm{nT}$, giữa Addis Ababa và Qsaybeh là $17 \mathrm{nT}$ (cùng tính cho ngày 3-1-2005).

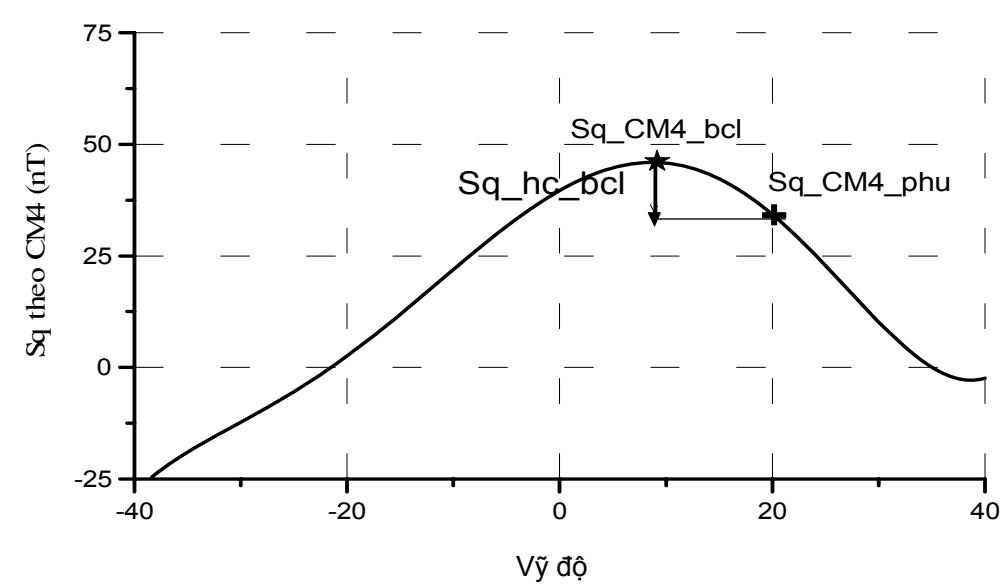

$\leftarrow$ Hình $1 b$. Biên độ Sq tính theo mô hình CM4 tại kinh tuyến qua Việt Nam
Như vậy, biên độ thành phần $\mathrm{H}$ chỉ do EEJ gây ra sau khi đã hiệu chỉnh ảnh hưởng của hệ dòng $\mathrm{Sq}$ thu được tại trạm Huancayo và tương ứng với các trạm khác, tại Addis Ababa, Bạc Liêu như sau:

$\Delta$ H_EEJ_(hua)_sq $=\Delta \mathrm{H}$ _EEJ_(hua) -Sq_hc_(hua)

$\Delta H \_E E J \_(a a e) \_s q=\Delta H \_E E J \_(a a e)-S q \_h c \_(a a e)$

$\Delta \mathrm{H} \_E E J \_(b c l) \_s q=\Delta H \_E E J \_(b c l)-S q \_h c \_(b c l)$
Trên hình $1 c$, chúng tôi biểu diễn giá trị trường từ thành phần $\mathrm{H}$ do $\mathrm{EEJ}$ gây ra tính tại các trạm HUA, AAE, BCL cho toàn bộ số liệu năm 2002 sau khi đã hiệu chỉnh ảnh hưởng của dòng $\mathrm{Sq}$, với giá trị lớn nhất thu được tại HUA là $128 \mathrm{nT}$, tại $\mathrm{AAE}$ là $99 \mathrm{nT}$, và tại $\mathrm{BCL}$ là $104 \mathrm{nT}$. Theo hình vẽ này, hầu như các đỉnh cực trị của $\mathrm{EEJ}$ đều trùng với giữa trưa địa phương. Tuy nhiên, khi tính toán EEJ từ số liệu CHAMP cùng vị trí các trạm, thì giá
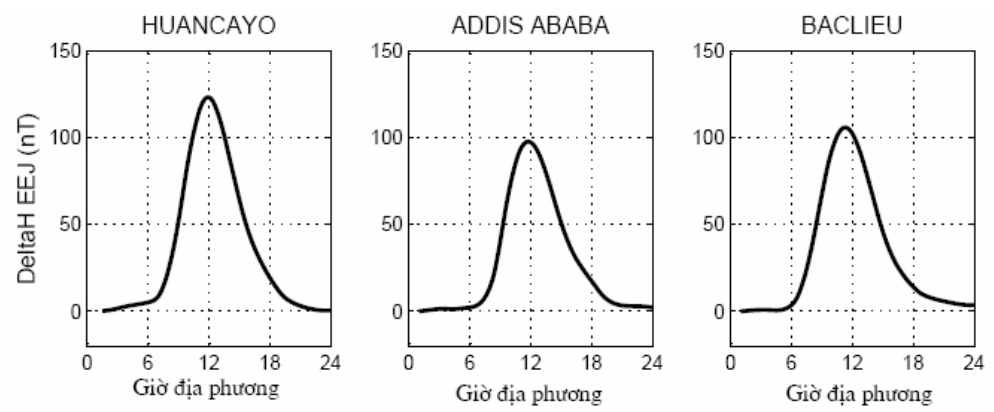

$\leftarrow$ Hình 1c. Giá trị trung bình giờ thành phần trường nằm ngang $\mathrm{H}$ do EEJ gây ra tính tại ba trạm (HUA, AAE, BCL) tính cho cả năm 2002 
trị thành phần $\mathrm{H}$ do EEJ gây ra tại HUA là 41nT, tại $\mathrm{AAE}$ là $29 \mathrm{nT}$ và tại $\mathrm{BCL}$ là $57 \mathrm{nT}$.

Sự chênh lệch giá trị thành phần $\mathrm{H}$ do EEJ gây ra tính từ hai loại số liệu trên tại cùng một vị trí là do:

- Khoảng cách từ quỹ đạo của vệ tinh CHAMP và các trạm quan sát đến tâm hệ dòng EEJ là khác nhau (vệ tinh CHAMP bay cách EEJ khoảng $300 \mathrm{~km}$ còn EEJ cách bề mặt trái đất khoảng $105 \mathrm{~km})$.

- Do ảnh hưởng của độ lệch giữa quỹ đạo vệ tinh và chiều của hệ dòng EEJ (kết quả về ảnh hưởng góc lệch này sẽ được công bố trong một nghiên cứu sau này), tuy nhiên ảnh hưởng này là không nhiều chỉ khoảng $5 \mathrm{nT}$ tuỳ theo các kinh tuyến khác nhau.

\subsection{Tính toán mật độ dòng tại tâm EEJ}

Khi ta coi EEJ như một bản dòng điện, chạy ổn định trên độ cao $105 \mathrm{~km}$ so với bề mặt trái đất, từ giá trị cường độ trường từ do $\mathrm{EEJ}$ (cả số liệu thu được trên vệ tinh, và số liệu các trạm trên mặt đất) gây ra ở trên áp dụng công thức được phát triển bởi Doumouya [5] ta tính được mật độ dòng $\mathrm{I}_{12}$ (Ampe/Km) tại tâm của $\mathrm{EEJ}$ vào giữa trưa địa phương như sau:

$$
I_{12}=\frac{\Delta H_{0}}{0.4 \operatorname{arctg}\left(\frac{a}{h}\right)}
$$

h: độ cao của EEJ (nếu lấy số liệu tại các trạm mặt đất thì $\mathrm{h}=105 \mathrm{~km}$, nếu lấy số liệu vệ tinh CHAMP thì $\mathrm{h}=284 \mathrm{~km}$ )

\section{$\mathrm{a}: 1 / 2$ bề rộng của EEJ}

$\Delta \mathrm{H}_{0}$ : giá trị thành phần nằm ngang của trường từ gây ra bởi EEJ.

\section{Kết quả tính toán và thảo luận}

Trên hình $2 a$, chúng tôi biểu diễn giá trị mật độ dòng của EEJ tính từ số liệu vệ tinh CHAMP tại giữa trưa địa phương cho toàn bộ sáu năm số liệu hiện có (từ 2002-2007) phụ thuộc vào kinh tuyến địa lý. Với mùa địa từ được quy định như sau: mùa hè gồm các tháng $5,6,7,8$, mùa đông: tháng 11 , $12,1,2$ và hai phân điểm là: xuân phân: tháng 3,4 ; thu phân: tháng 9,10 . Hình $2 b$, biểu diễn mật độ dòng do EEJ gây ra tính được từ số liệu của ba cặp trạm với các mùa và thời gian tương ứng với hình 2a. Trên các hình này, chúng ta nhận thấy rằng:

- Vào mùa hè và hai phân điểm, biên độ EEJ có xu hướng biến thiên khá giống nhau nó đều tồn tại bốn đỉnh cực đại trên toàn kinh tuyến tại các kinh tuyến có vị trí: $105^{\circ} \mathrm{E}, 0^{\circ}, 90^{\circ} \mathrm{W}, 180^{\circ} \mathrm{W}$ và bốn điểm cực tiểu tại vị trí: $45^{\circ} \mathrm{E}, 140^{\circ} \mathrm{E}, 40^{\circ} \mathrm{W}, 135^{\circ} \mathrm{W}$. Đỉnh cực đại tại kinh tuyến $105^{\circ} \mathrm{E}$ có biên độ lớn nhất. Sự xuất hiện bốn đỉnh dị thường này đã được England [7] phát hiện khi nghiên cứu về trường điện trên lớp $\mathrm{E}$ của tầng điện ly và gọi là "số sóng bội 4" của EEJ. Nó xuất hiện hàng ngày và trong khoảng thời gian giữa trưa địa phương. Khi so sánh EEJ thu được tại vị trí của ba trạm (bảng 2) thì EEJ tính tại HUA lại lớn hơn tại BCL trong các tháng phân điểm và có biên độ bằng nhau trong mùa hè, điều này có thể là do ảnh hưởng của góc lệch giữa quỹ đạo vệ tinh CHAMP và hướng của hệ dòng EEJ tại HUA lớn nên EEJ tinh từ số liệu CHAMP đã bị giảm đi.

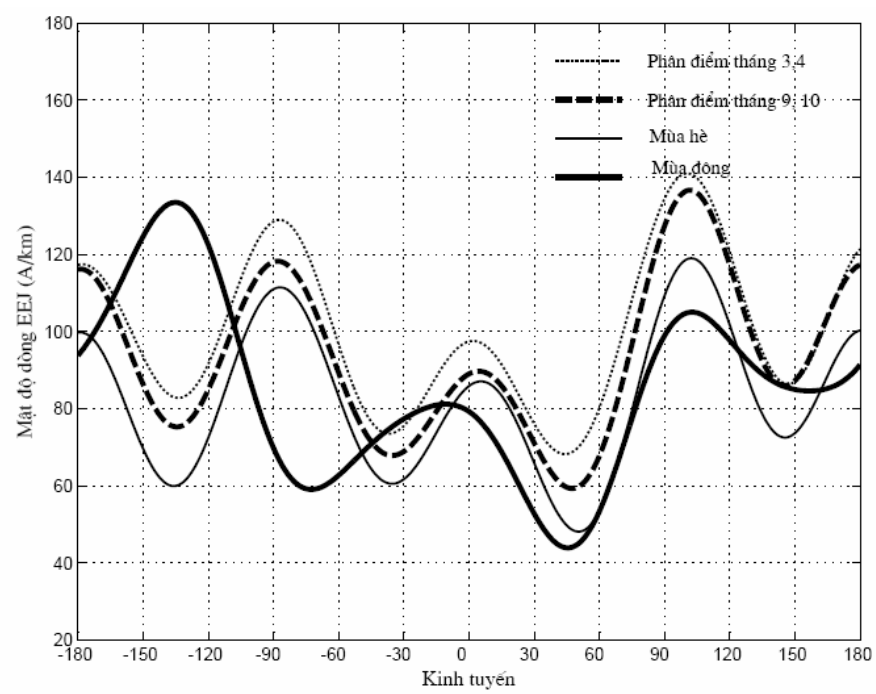

$\leftarrow$ Hình $2 a$. Biến thiên theo mùa của EEJ từ số liệu vệ tinh CHAMP với sáu năm số liệu (2002-2007) 
$\rightarrow$ Hình $2 b$. Biến thiên theo mùa của EEJ từ số liệu ba trạm mặt đất với sáu năm số liệu (2002-2007) với đường đậm nét liền là mùa đông, đường mảnh nét liền là mùa hè, đường mảnh nét rời là phân điểm tháng 3,4 và đường đậm nét rời là phân điểm tháng 9,10
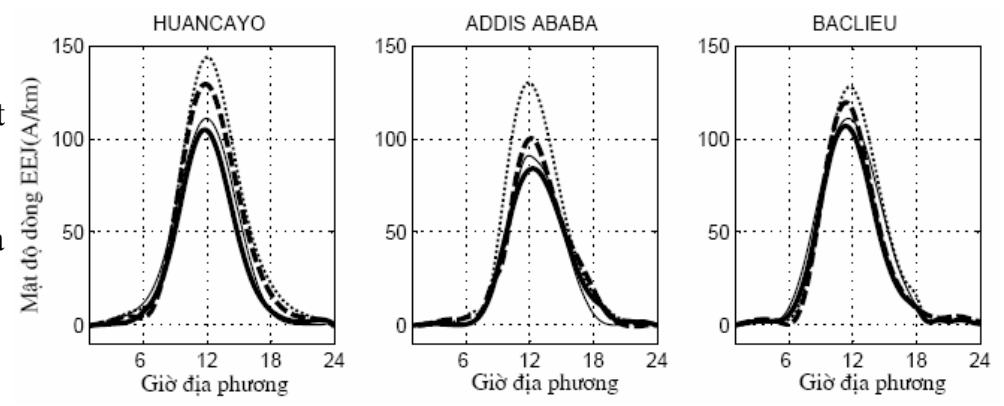

Bảng 2. Giá trị mật độ dòng của EEJ tại vị trí các đài trạm tại các mùa

\begin{tabular}{|c|c|c|c|c|c|c|}
\hline \multirow[b]{2}{*}{ Mùa } & \multicolumn{2}{|c|}{ EEJ tại HUA (A/km) } & \multicolumn{2}{|c|}{ EEJ tại AAE (A/km) } & \multicolumn{2}{|c|}{ EEJ tại BCL (A/km) } \\
\hline & $\begin{array}{l}\text { Tính từ } \\
\text { đài trạm }\end{array}$ & Tính từ CHAMP & $\begin{array}{l}\text { Tính từ } \\
\text { đài trạm }\end{array}$ & Tính từ CHAMP & $\begin{array}{l}\text { Tính từ } \\
\text { đài trạm }\end{array}$ & Tính từ CHAMP \\
\hline Xuân phân & 144.0 & 122.2 & 130.2 & 69.8 & 128.0 & 139.7 \\
\hline Thu phân & 129.3 & 111.5 & 100.2 & 62.4 & 119.5 & 135.9 \\
\hline Mùa hè & 110.9 & 105.6 & 91.0 & 54.5 & 110.8 & 118.5 \\
\hline Mùa đông & 104.8 & 59.4 & 84.2 & 45.6 & 106.8 & 104.8 \\
\hline
\end{tabular}

- Biến thiên của $E E J$ vào các tháng mùa đông thì lại có xu hướng khác với các mùa khác, nó chỉ xuất hiện ba cực đại tại $135^{\circ} \mathrm{W}, 10^{\circ} \mathrm{W} 100^{\circ} \mathrm{E}$ và ba cực tiểu tại: $75^{\circ} \mathrm{W}, 45^{\circ} \mathrm{E}, 160^{\circ} \mathrm{E}$, hay còn gọi là "số sóng bội 3". Trong đó, cực đại tại $135^{\circ} \mathrm{W}$ là mạnh nhất. Trong nghiên cứu của Hagan [8] sự xuất hiện chỉ ba cực trị trong mùa đông có thể là do ảnh hưởng của gió mặt trời hoạt động yếu trong mùa đông, khi kết hợp với các hệ dòng tại lớp $\mathrm{E}$ tầng điện $l y$, nó làm dịch chuyển các đỉnh dị thường và chỉ còn xuất hiện "số sóng bội 3".

- Biên độ của EEJ theo từng mùa tính từ cả hai loại số liệu trên đều phản ánh qui luật: EEJ lớn nhất vào xuân phân và giảm dần vào thu phân, mùa hè, yếu nhất vào mùa đông. Trong [19], Tarpley đã giải thích hiện tượng này bằng giả thiết là do sự thay đổi tâm của hệ dòng $\mathrm{Sq}$, trong các tháng phân điểm cả ở bán cầu bắc và bán cầu nam, tâm này có xu hướng dịch chuyển về xích đạo, còn vào các tháng mùa hè hay mùa đông các tâm này dịch chuyển về phía cực. Khi đó, do sự ảnh hưởng tổng hợp của của hệ dòng $\mathrm{Sq}$, biên độ của $\mathrm{EEJ}$ vào các tháng phân điểm sẽ lớn hơn. Riêng khu vực kinh tuyến từ $110^{\circ} \mathrm{W}-180^{\circ} \mathrm{W}, \mathrm{EEJ}$ tính từ số liệu CHAMP vào mùa đông lại mạnh lên đáng kể tuy nhiên do không có số liệu của đài trạm trong khu vực này nên chưa so sánh được. Chúng ta cũng cần sử dụng thêm số liệu khác (như GPS, điện ly,...) để chứng minh sự tồn tại bốn đỉnh dị thường trên toàn cầu. Cũng cần chú ý rằng trong bài báo này do còn hạn chế về số liệu thu được tại các trạm dưới mặt đất chúng tôi mới sử dụng số liệu của các trạm nằm về phía bắc bán cầu mà chưa có số liệu phía nam bán cầu để so sánh.

- Trong bảng 2 là kết quả tính toán giá trị mật độ dòng điện của EEJ thu được tại vị trí ba trạm tính từ số liệu vệ tinh $\mathrm{CHAMP}$ và từ các trạm cho các mùa khác nhau. Chúng đều lớn nhất vào xuân phân và nhỏ nhất vào mùa đông. Tuy nhiên, tại vị trí của trạm AAE giá trị EEJ thu được giữa hai loại số liệu trên lệch nhau nhiều, điều này có thể là do góc lệch giữa quỹ đạo của vệ tinh CHAMP và chiều của dòng EEJ qua khu vực này lớn hơn các khu vực của hai cặp trạm còn lại.

- Hình $3 a$ biểu diễn giá trị mật độ dòng EEJ phụ thuộc vào kinh tuyến vào mùa hè của các năm 2002, 2004, 2007 thu được trên vệ tinh CHAMP, hình $3 b$ là mật độ $\mathrm{EEJ}$ tính từ số liệu của ba trạm trên mặt đất với thời gian tương ứng và hình $3 \mathrm{c}$ trình bày giá trị trung bình tháng số vết đen mặt trời thu được trong cùng khoảng thời gian. Theo đó, vào những năm mặt trời hoạt động mạnh hơn như năm 2002-2003 khi số vết đen mặt trời lớn thì mật độ dòng EEJ thu được cũng lớn hơn, vào năm 2006-2007 khi mặt trời hoạt động yếu thì EEJ cũng yếu hơn. Tuy nhiên chúng ta cần tiếp tục nghiên cứu quy luật biến đổi theo thời gian của EEJ cho những năm tiếp theo với ít nhất là một chu kỳ hoạt động của Mặt trời trên cả hai loại số liệu để khẳng định quy luật trên, nhất là hiện nay vệ tinh CHAMP vẫn đang hoạt động và cho số liệu đều đặn. 


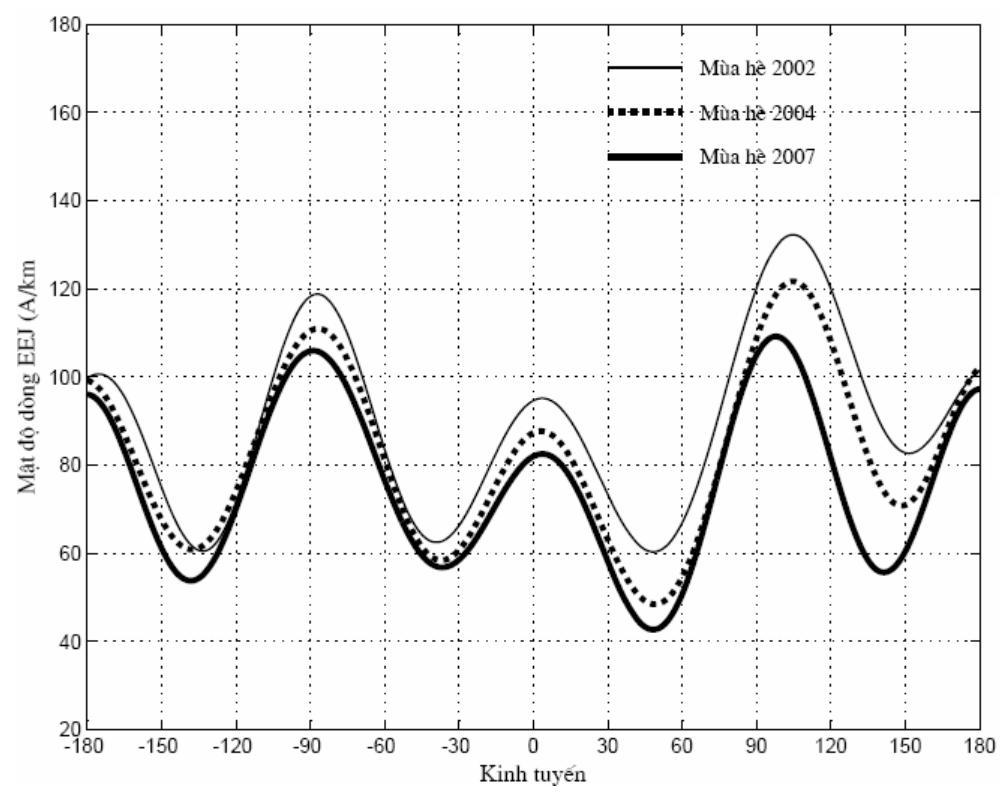

$\leftarrow$ Hình $3 a$. EEJ vào mùa hè tính từ số liệu vệ tinh CHAMP năm 2002, 2004, 2007

$\rightarrow$ Hìn $3 b$. EEJ vào mùa hè tính từ số liệu ba tram từ năm 2002 đến 2007
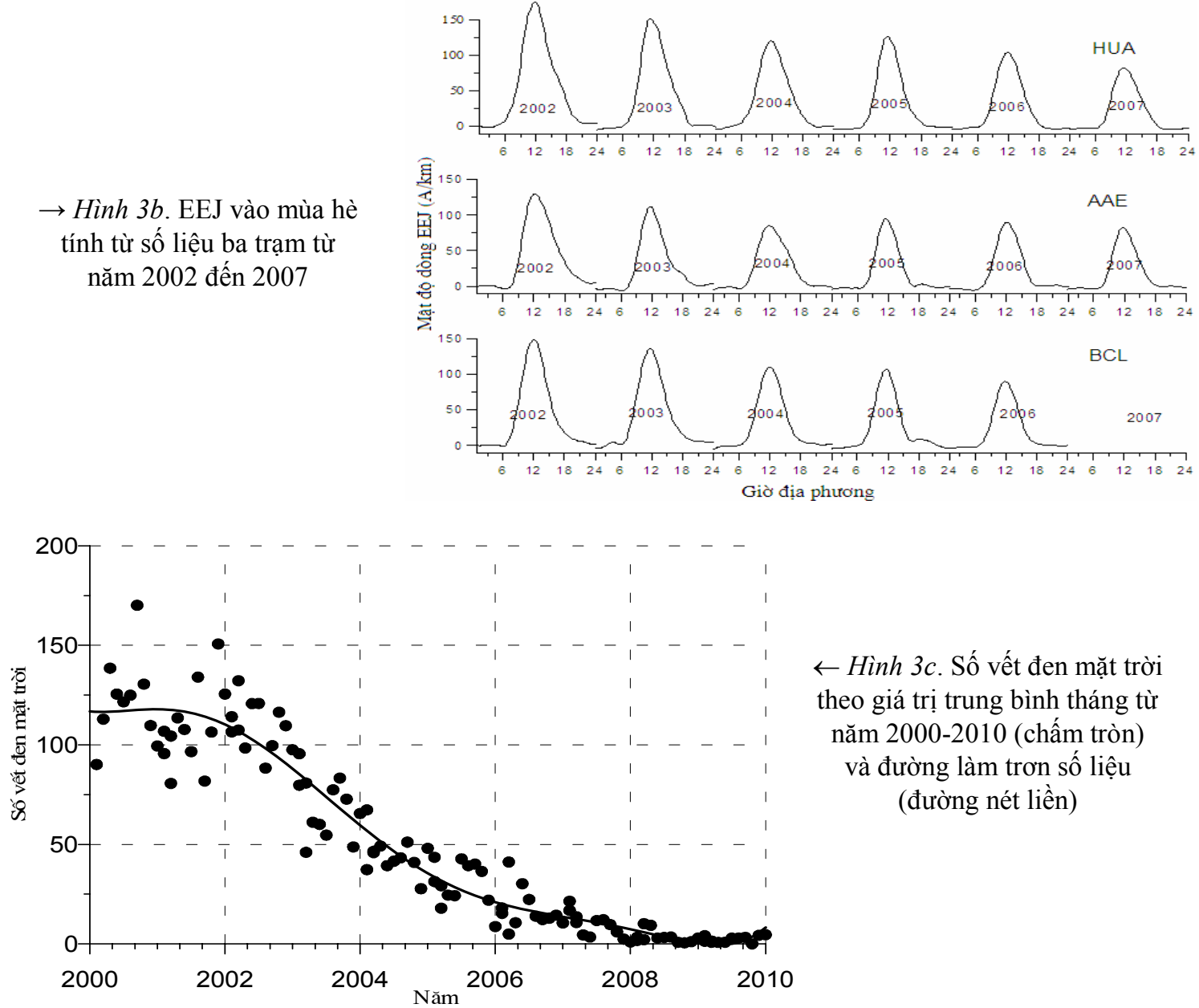

$\leftarrow$ Hình $3 c$. Số vết đen mặt trời theo giá trị trung bình tháng từ năm 2000-2010 (chấm tròn) và đường làm trơn số liệu (đường nét liền) 


\section{Kết luận}

Sau khi tính toán và nghiên cứu quy luật biến thiên theo thời gian của $\mathrm{EEJ}$ dọc theo các kinh tuyến khác nhau, tính từ số liệu thu được trên vệ tinh CHAMP trong vòng nửa chu kỳ hoạt động của mặt trời cũng như từ số liệu thu được tại các đài trạm mặt đất chúng tôi rút ra một số kết luận quan trọng sau:

- Mật độ dòng của EEJ các tháng mùa hè và và các tháng phân điểm tồn tại bốn đỉnh cực đại và bốn đỉnh cực tiểu EEJ trên toàn cầu, trong đó đỉnh cực trị qua Việt $\operatorname{Nam}\left(105^{\circ} \mathrm{E}\right)$ là mạnh nhất so với các kinh tuyến khác. Trong mùa đông, EEJ chỉ tồn tại ba đỉnh cực đại và ba đỉnh cực tiểu, cực đại qua kinh tuyến $135^{\circ} \mathrm{W}$ là lớn nhất.

- Biên độ của EEJ biến thiên tỷ lệ với số vết đen Mặt trời. Với năm Mặt trời hoạt động mạnh thì mật độ dòng của EEJ lớn hơn những năm Mặt trời hoạt động yếu.

- Các biến thiên theo mùa của $\mathrm{EEJ}$ tính từ số liệu vệ tinh và số liệu các đài trạm khá tương ứng với nhau, chúng cùng mạnh lên vào các tháng phân điểm, mạnh nhất vào xuân phân và yếu đi vào mùa hè, yếu nhất vào mùa đông (trừ $\mathrm{khu}$ vực trong khoảng từ kinh tuyến $120^{\circ} \mathrm{W}$ đến $150^{\circ} \mathrm{W}, \mathrm{EEJ}$ vào mùa đông lại lớn hơn vào mùa hè).

Mối tương quan của quy luật biến đổi của EEJ theo mùa giữa hai loại số liệu là rất tốt, như vậy có thể khẳng định số liệu trường từ thu được trên vệ tinh CHAMP là chuỗi số liệu rất quý để nghiên cứu về EEJ trên toàn cầu, điều mà việc sử dụng số liệu thu được tại các trạm không phản ánh được do sự phân bố quá ít dọc theo tâm của EEJ.

Tuy nhiên, việc tính phần ảnh hưởng của EEJ từ số liệu vệ tinh CHAMP vẫn còn bị ảnh hưởng của nhiều nguồn khác mà chúng ta vẫn chưa loại trừ hết được, nhưng đây vẫn là chuỗi số liệu rất quan trọng để chúng ta nghiên cứu về biến thiên của EEJ theo thời gian trên toàn cầu.

Lời cảm ơn: Các tác giả bày tỏ lòng cám ơn TS. Doumouya đã có những ý kiến đóng góp xác đáng trong việc hoàn thành bài báo này. Bài báo được hoàn thành với phần số liệu vệ tinh được cung cấp bởi Viện Vật lý địa cầu Paris trong chương trình hợp tác giữa Viện Vật lý địa cầu - Hà Nội, Viện Vật lý địa cầu - Paris và Trung tâm nghiên cứu môi trường Trái Đất và các Hành tinh-
"Nghiên cứu Vật lý địa cầu trong mối quan hệ Mặt trời - Trái Đất, nghiên cứu trường từ ở Việt Nam" (PICS-3366) và kinh phí của các đề tài nghiên cứu cơ bản mã số: 105.01.42.09 và 105.99.74.09. Các tác giả cũng cảm ơn các ý kiến đóng góp và các trung tâm số liệu địa từ khác đã cung cấp số liệu tại các đài trạm để hoàn thiện bài báo.

\section{TÀI LIẸU DÃNN}

[1] Agu C.E., Onwumechili C.A., 1981: Comparison of the POGO satellite and ground measurement of the magnetic field of the equatorial electrojet, J. Atmos. and Terr Phys, 43, 801-807.

[2] Arora B.R., Mahahasbde M.V., 1993: Indian IEEY geomagnetic observational program and some preliminary results, J. Geophys, 11, 365-384.

[3] Chandra H., Sinha H.S.S and Rastogi R.G., 2000: Equatorial electrojet studies from rocket and ground measurements, Earth Planets Space, 52, 111-120.

[4] Chapman S., 1951: The equatorial electrojet as detected from the abnormal electric current distributions above Huancayo, Peru and elsewhere, Arch. Meteorol. Geophys. Bioclimatol. A4, 368-390.

[5] Doumouya V., Vassal J., Cohen Y., Fambitakyoye $O$. and Menvielle M., 1998: Equatorial electrojet at African longitudes: first results from magnetic measurements, Ann. Geophysics, 16, 658-676.

[6] Doumouya V., Cohen Y., 2004: Improving and testing the empirical equatorial electrojet model with CHAMP satellite data, Ann. Geophysics, 3323-3333.

[7] England S.L., 2006: Longitudinal variation of the E-region electric fields caused by atmospheric tides, Geophys.Res.Lett., 33.

[8] Hagan M.E. and Forbes J.M., 2002: Migrating and nonmigrating diurnal tides in the middle and upper atmosphere excited by tropospheric latent heat release, Geophys.Res., 107(D24), 4754, doi:10.1029/2001JD001236.

[9] Kim H.R., King D., 1999: A study of local time and longitudinal variability of the amplitude of the equatorial electrojet observed in POGO satellite data, Earth Planets Space, 51, 373-381. 
[10] Le Mouël J.L., Shebalin P. and Chulliat $A$., 2005: The field of the equatorial electrojet from CHAMP data, Ann. Geophysics, 14, 38-46.

[11] Onwumechili C. A., 1985: Satellite measurements of the equatorial electrojet, $\mathrm{J}$. Geomag. Geoelectr., 37, 11-36.

[12] Onwumechili C. A. and Agu C. E., 1980: General features of the magnetic field of the equatorial electrojet measured by the POGO satellites, Planet. Space Sci., 28, 1125-1130.

[13] Rabiu A.B., Mamukuyomi A.I., and Joshua E.O., 2007: Variability of equatorial ionosphere inferred from geomagnetic field measurements, Earth Planets Space, 65, 607-618.

[14] Rastogi R.G., 1989: The equatorial electrojet in magnetic and ionospheric effects, Geomagnetism, 3, 461-525.

[15] Rangarajan G.K., 1973: Indices of Geomagnetic Activity, In Geomagnetism, Vol. 3 Edited by J.A. Jacobs.
[16] Rao D.R.K, Hao T.Q., Thoa N.T.K., 1997: Comparative study of the Equatorial Electrojet Strengths in the Indian and Vietnamese sectors during IEEY. IAGA/UPPSALA, August 4th., 1997.

[17] Patric A., Stefan M., 2002: Spatio-temporal characterization of the equatorial electrojet from CHAMP, Orsted, and SAC-C satellite magnetic measurements, Geophys. J. Int., 112, 1-10.

[18] Sabaka J., Olsen T.N., 2004: Extending comprehensive models of the Earth's magnetic field with Orsted CHAMP data, Geophys. J. Int., 159, 521-547.

[19] Tarpley J.D., 1973: Seasonal movement of the sq current foci and related effects in the equatorial electrojet, Terr Phys, 35, 101-107.

[20] Lê Truòng Thanh, Doumouya VafI, Lê Huy Minh và Hà Duyên Châu, 2010: Mô hình dòng điện xích đạo từ số liệu vệ tinh CHAMP, Tạp chí Các khoa học về Trái Đất, T. 32, 1, 48-56.

\section{SUMMARY}

\section{Anomaly of equatorial electrojet (EEJ) and its seasonal variation}

In this paper we apply the new technique to separate the effect of the EEJ with the magnetic data received on CHAMP satellite from 2002 to 2007 to study the anomaly of the EEJ and its season variation in global scale. We found that: In summer and equinox, along the magnetic equator there are 4 maximum peaks and 4 minima of the current density of the EEJ. These maximum peaks are at the longitudes: $105^{\circ} \mathrm{E}, 0^{\circ}, 90^{\circ} \mathrm{W}$ and $180^{\circ} \mathrm{W}$, among which the peak at the Vietnamese sector $\left(105^{\circ} \mathrm{E}\right)$ is maximal. These minima are at the longitudes $45^{\circ} \mathrm{E}, 140^{\circ} \mathrm{E}, 40^{\circ} \mathrm{W}$ and $135^{\circ} \mathrm{W}$. In winter, the EEJ have only 3 peaks at $135^{\circ} \mathrm{W}, 10^{\circ} \mathrm{W}$, and $100^{\circ} \mathrm{E}$. The peak at $135^{\circ} \mathrm{W}$ is maximal. The current density of the EEJ is maximum in the equinox, smaller in the summer and smallest in the winter. In the period when the Sun is active, the current density of the EEJ is bigger than that in the calm period of the Sun. Additionally, these results are compared with those obtained from the magnetic data received at the 3 observatories pairs on the Earth's surface with one of the observatories in the immediate proximity to the dip-equator (HUA, AAE, BCL) and the other outside of the EEJ footprint area (FUQ, QSB, PHU) for consolidating the well-founded properties and the magnetic data received on CHAMP are very useful to study the EEJ in Vietnam and over the world. 